\title{
Rational exponents in extremal graph theory
}

\author{
Boris Bukh* David Conlon ${ }^{\dagger}$
}

\begin{abstract}
Given a family of graphs $\mathcal{H}$, the extremal number $\operatorname{ex}(n, \mathcal{H})$ is the largest $m$ for which there exists a graph with $n$ vertices and $m$ edges containing no graph from the family $\mathcal{H}$ as a subgraph. We show that for every rational number $r$ between 1 and 2, there is a family of graphs $\mathcal{H}_{r}$ such that $\operatorname{ex}\left(n, \mathcal{H}_{r}\right)=\Theta\left(n^{r}\right)$. This solves a longstanding problem in the area of extremal graph theory.
\end{abstract}

\section{Introduction}

Given a family of graphs $\mathcal{H}$, another graph $G$ is said to be $\mathcal{H}$-free if it contains no graph from the family $\mathcal{H}$ as a subgraph. The extremal number $\operatorname{ex}(n, \mathcal{H})$ is then defined to be the largest number of edges in an $\mathcal{H}$-free graph on $n$ vertices. If $\mathcal{H}$ consists of a single graph $H$, the classical Erdös-Stone-Simonovits theorem [9, 10] gives a satisfactory first estimate for this function, showing that

$$
\operatorname{ex}(n, H)=\left(1-\frac{1}{\chi(H)-1}+o(1)\right)\left(\begin{array}{l}
n \\
2
\end{array}\right)
$$

where $\chi(H)$ is the chromatic number of $H$.

When $H$ is bipartite, the estimate above shows that $\operatorname{ex}(n, H)=o\left(n^{2}\right)$. This bound is easily improved to show that for every bipartite graph $H$ there is some positive $\delta$ such that $\operatorname{ex}(n, H)=O\left(n^{2-\delta}\right)$. However, there are very few bipartite graphs for which we have matching upper and lower bounds.

The most closely studied case is when $H=K_{s, t}$, the complete bipartite graph with parts of order $s$ and $t$. In this case, a famous result of Kővári, Sós and Turán [15] shows that ex $\left(n, K_{s, t}\right)=O_{s, t}\left(n^{2-1 / s}\right)$ whenever $s \leq t$. This bound was shown to be tight for $s=2$ by Esther Klein [6] (see also [3, 8]) and for $s=3$ by Brown [3]. For higher values of $s$, it is only known that the bound is tight when $t$ is sufficiently large in terms of $s$. This was first shown by Kollár, Rónyai and Szabó [14, though their construction was improved slightly by Alon, Rónyai and Szabó [1], who showed that there are graphs with $n$ vertices and $\Omega_{s}\left(n^{2-1 / s}\right)$ edges containing no copy of $K_{s, t}$ with $t=(s-1) !+1$.

Alternative proofs showing that $\operatorname{ex}\left(n, K_{s, t}\right)=\Omega_{s}\left(n^{2-1 / s}\right)$ when $t$ is significantly larger than $s$ were later found by Blagojević, Bukh and Karasev [2] and by Bukh [4]. In both cases, the basic idea behind the construction is to take a random polynomial $f: \mathbb{F}_{q}^{s} \times \mathbb{F}_{q}^{s} \rightarrow \mathbb{F}_{q}$ and then to consider the graph $G$ between two copies of $\mathbb{F}_{q}^{s}$ whose edges are all those pairs $(x, y)$ such that $f(x, y)=0$. A further application of this random algebraic technique was recently given by Conlon [5], who showed that for

\footnotetext{
*Department of Mathematical Sciences, Carnegie Mellon University, Pittsburgh, PA 15213, USA. E-mail: bbukh@math.cmu.edu. Research supported in part by a Sloan Research Fellowship, NSF grant DMS-1301548, and NSF CAREER grant DMS-1555149.

${ }^{\dagger}$ Mathematical Institute, Oxford OX2 6GG, United Kingdom. E-mail: david.conlon@maths.ox.ac.uk. Research supported by a Royal Society University Research Fellowship and ERC Starting Grant 676632.
} 

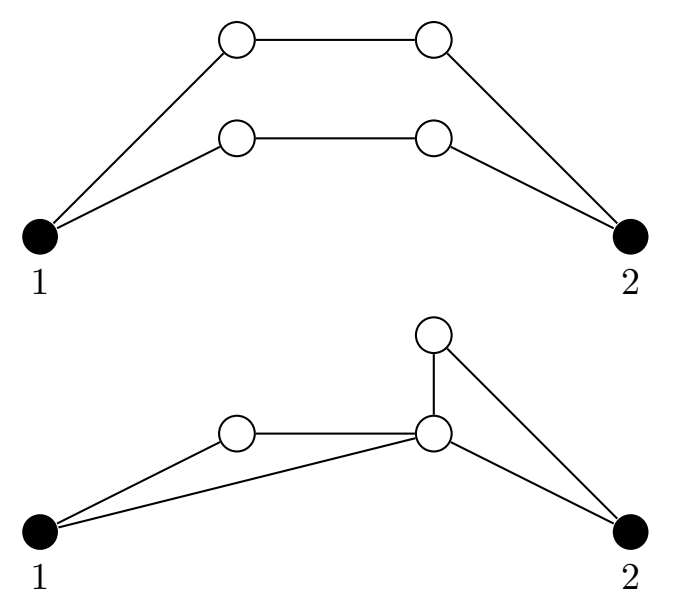
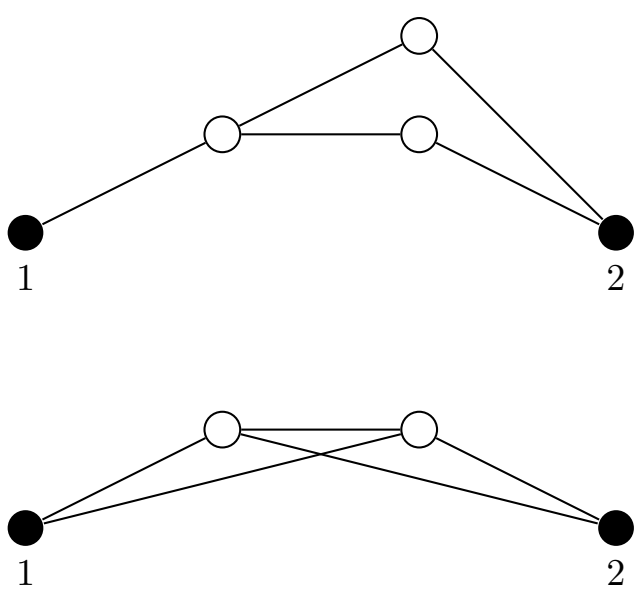

Figure 1: Some of the graphs in $\mathcal{T}^{2}$ when $(T, R)$ is a path of length 3 with rooted endpoints. The remaining graphs in $\mathcal{T}^{2}$ are obtained by swapping the two roots, which are labelled 1 and 2 .

every natural number $k \geq 2$ there exists a natural number $\ell$ such that, for every $n$, there is a graph on $n$ vertices with $\Omega_{k}\left(n^{1+1 / k}\right)$ edges for which there are at most $\ell$ paths of length $k$ between any two vertices. By a result of Faudree and Simonovits [11, this is sharp up to the implied constant. We refer the interested reader to [5] for further background and details.

In this paper, we give yet another application of the random algebraic method, proving that for every rational number between 1 and 2 , there is a family of graphs $\mathcal{H}_{r}$ for which $\operatorname{ex}\left(n, \mathcal{H}_{r}\right)=\Theta\left(n^{r}\right)$. This solves a longstanding open problem in extremal graph theory that has been reiterated by a number of authors, including Frankl [12] and Füredi and Simonovits [13].

Theorem 1.1 For every rational number $r$ between 1 and 2 , there exists a family of graphs $\mathcal{H}_{r}$ such that ex $\left(n, \mathcal{H}_{r}\right)=\Theta\left(n^{r}\right)$.

Prior to our work, the main result in this direction was due to Frankl [12, who showed that for any rational number $r \geq 1$ there exists a family of $k$-uniform hypergraphs whose extremal function is $\Theta\left(n^{r}\right)$. However, in Frankl's work, the uniformity $k$ depends on the desired exponent $r$, whereas we can always take $k=2$.

In order to define the relevant families $\mathcal{H}_{r}$, we need some preliminary definitions.

Definition 1.1 A rooted tree $(T, R)$ consists of a tree $T$ together with an independent set $R \subset V(T)$, which we refer to as the roots. When the set of roots is understood, we will simply write $T$.

Each of our families $\mathcal{H}_{r}$ will be of the following form.

Definition 1.2 Given a rooted tree $(T, R)$, we define the pth power $\mathcal{T}_{R}^{p}$ of $(T, R)$ to be the family of graphs consisting of all possible unions of $p$ distinct labelled copies of $T$, each of which agree on the set of roots $R$. Again, we will usually omit $R$, denoting the family by $\mathcal{T}^{p}$ and referring to it as the pth power of $T$. 

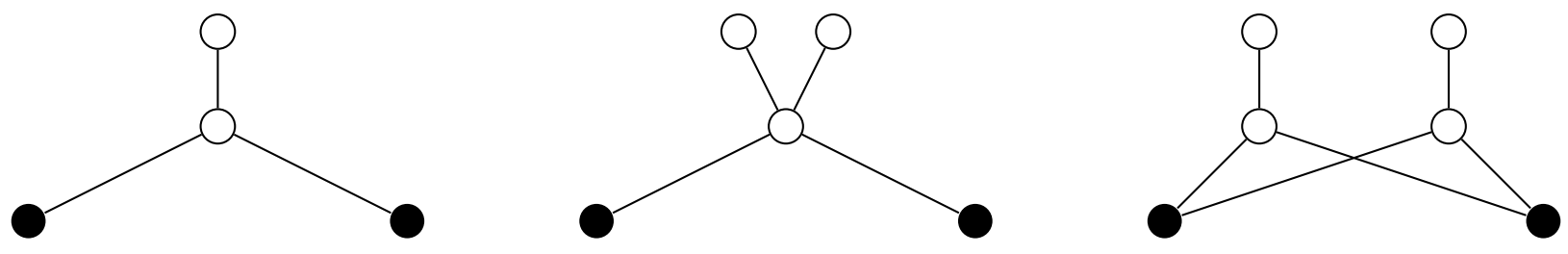

Figure 2: An unbalanced rooted tree $T$ and two elements of $\mathcal{T}^{2}$.

We note that $\mathcal{T}^{p}$ consists of more than one graph because we allow the unrooted vertices $V(T) \backslash R$ to meet in every possible way. For example, if $T$ is a path of length 3 whose endpoints are rooted, the family $\mathcal{T}^{2}$ contains a cycle of length 6 and the various degenerate configurations shown in Figure 1 . The following parameter will be critical in studying the extremal number of the family $\mathcal{T}^{p}$.

Definition 1.3 Given a rooted tree $(T, R)$, we define the density $\rho_{T}$ of $(T, R)$ to be $\frac{e(T)}{v(T)-|R|}$.

The upper bound in Theorem 1.1 will follow from an application of the next lemma.

Lemma 1.1 For any rooted tree $(T, R)$ with at least one root, the family $\mathcal{T}^{p}$ satisfies

$$
e x\left(n, \mathcal{T}^{p}\right)=O_{p}\left(n^{2-1 / \rho_{T}}\right) .
$$

It would be wonderful if there were also a matching lower bound for $\operatorname{ex}\left(n, \mathcal{T}^{p}\right)$. However, this is in general too much to expect. If, for example, $(T, R)$ is the star $K_{1,3}$ with two rooted leaves, $\mathcal{T}^{2}$ will contain the graph shown in Figure 2 where the two central vertices agree. However, this graph is a tree, so it is easy to show that $\operatorname{ex}\left(n, \mathcal{T}^{2}\right)=O(n)$, whereas, since $\rho_{T}=3 / 2$, Lemma 1.1 only gives $\operatorname{ex}\left(n, \mathcal{T}^{2}\right)=O\left(n^{4 / 3}\right)$. Luckily, we may avoid these difficulties by restricting attention to so-called balanced trees.

Definition 1.4 Given a subset $S$ of the unrooted vertices $V(T) \backslash R$ in a rooted tree $(T, R)$, we define the density $\rho_{S}$ of $S$ to be $e(S) /|S|$, where $e(S)$ is the number of edges in $T$ with at least one endpoint in $S$. Note that when $S=V(T) \backslash R$, this agrees with the definition above. We say that the rooted tree $(T, R)$ is balanced if, for every subset $S$ of the unrooted vertices $V(T) \backslash R$, the density of $S$ is at least the density of $T$, that is, $\rho_{S} \geq \rho_{T}$. In particular, if $|R| \geq 2$, then this condition guarantees that every leaf in the tree is a root.

With the caveat that our rooted trees must be balanced, we may now prove a lower bound matching Lemma 1.1 by using the random algebraic method.

Lemma 1.2 For any balanced rooted tree $(T, R)$, there exists a positive integer $p$ such that the family $\mathcal{T}^{p}$ satisfies

$$
e x\left(n, \mathcal{T}^{p}\right)=\Omega\left(n^{2-1 / \rho_{T}}\right)
$$

Therefore, given a rational number $r$ between 1 and 2, it only remains to identify a balanced rooted tree $(T, R)$ for which $2-1 / \rho_{T}$ is equal to $r$. 

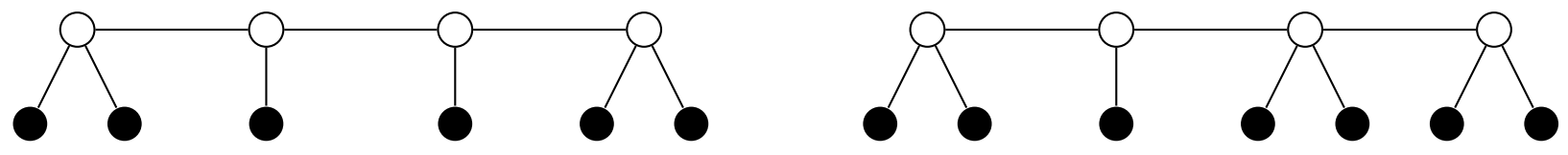

Figure 3: The rooted trees $T_{4,9}$ and $T_{4,10}$.

Definition 1.5 Suppose that $a$ and $b$ are natural numbers satisfying $a-1 \leq b<2 a-1$ and put $i=b-a$. We define a rooted tree $T_{a, b}$ by taking a path with a vertices, which are labelled in order as $1,2, \ldots, a$, and then adding an additional rooted leaf to each of the $i+1$ vertices

$$
1,\left\lfloor 1+\frac{a}{i}\right\rfloor,\left\lfloor 1+2 \cdot \frac{a}{i}\right\rfloor, \ldots,\left\lfloor 1+(i-1) \cdot \frac{a}{i}\right\rfloor, a .
$$

For $b \geq 2 a-1$, we define $T_{a, b}$ recursively to be the tree obtained by attaching a rooted leaf to each unrooted vertex of $T_{a, b-a}$.

Note that the tree $T_{a, b}$ has $a$ unrooted vertices and $b$ edges, so that $\rho_{T}=b / a$. Now, given a rational number $r$ with $1<r<2$, let $a / b=2-r$ and let $\mathcal{T}_{a, b}^{p}$ be the $p$ th power of $T_{a, b}$. To prove Theorem 1.1. it will suffice to prove that $T_{a, b}$ is balanced, since we may then apply Lemmas 1.1 and 1.2 to $\mathcal{T}_{a, b}^{p}$, for $p$ sufficiently large, to conclude that

$$
\operatorname{ex}\left(n, \mathcal{T}_{a, b}^{p}\right)=\Theta\left(n^{2-a / b}\right)=\Theta\left(n^{r}\right)
$$

Therefore, the following lemma completes the proof of Theorem 1.1 .

Lemma 1.3 The tree $T_{a, b}$ is balanced.

All of the proofs will be given in the next section: we will prove the easy Lemma 1.1 in Section 2.1; Lemma 1.3 and another useful fact about balanced trees will be proved in Section 2.2. and Lemma 1.2 will be proved in Section 2.3. We conclude, in Section 3, with some brief remarks.

\section{Proofs}

\subsection{The upper bound}

We will use the following folklore lemma.

Lemma 2.1 A graph $G$ with average degree d has a subgraph $G^{\prime}$ of minimum degree at least d/2.

With this mild preliminary, we are ready to prove Lemma 1.1, that $\operatorname{ex}\left(n, \mathcal{T}^{p}\right)=O_{p}\left(n^{2-1 / \rho_{T}}\right)$ for any rooted tree $(T, R)$.

Proof of Lemma 1.1: Suppose that $G$ is a graph on $n$ vertices with $c n^{2-\alpha}$ edges, where $\alpha=1 / \rho_{T}$ and $c \geq 2 \max (|T|, p)$. We wish to show that $G$ contains an element of $\mathcal{T}^{p}$. Since the average degree of $G$ is $2 c n^{1-\alpha}$, Lemma 2.1 implies that $G$ has a subgraph $G^{\prime}$ with minimum degree at least $c n^{1-\alpha}$. 
Suppose that this subgraph has $s \leq n$ vertices. By embedding greedily one vertex at a time, the minimum degree condition allows us to conclude that $G^{\prime}$ contains at least

$$
s \cdot c n^{1-\alpha} \cdot\left(c n^{1-\alpha}-1\right) \cdots\left(c n^{1-\alpha}-|T|+2\right) \geq(c / 2)^{|T|-1} s n^{(|T|-1)(1-\alpha)}
$$

labelled copies of the (unrooted) tree $T$. Since there are at most $s^{|R|}$ possible choices for the root vertices $R$, there must be some choice $R_{0}$ for these vertices in at least

$$
\frac{(c / 2)^{|T|-1} s n^{(|T|-1)(1-\alpha)}}{s^{|R|}} \geq \frac{(c / 2)^{|T|-1} n^{(|T|-1)(1-\alpha)}}{n^{|R|-1}}=(c / 2)^{|T|-1}
$$

distinct labelled copies of $T$, where we used that $s \leq n$ and $\alpha=1 / \rho_{T}=(|T|-|R|) /(|T|-1)$. Since $(c / 2)^{|T|-1} \geq p$, this gives the required element of $\mathcal{T}^{p}$.

\section{$2.2 \quad$ Balanced trees}

We will begin by proving Lemma 1.3, that $T_{a, b}$ is balanced.

Proof of Lemma 1.3: Suppose that $S$ is a proper subset of the unrooted vertices of $T_{a, b}$. We wish to show that $e(S)$, the number of edges in $T$ with at least one endpoint in $S$, is at least $\rho_{T}|S|$, where $\rho_{T}=b / a$. We may make two simplifying assumptions. First, we may assume that $a-1 \leq b<2 a-1$. Indeed, if $b \geq 2 a-1$, then the bound for $T_{a, b}$ follows from the bound for $T_{a, b-a}$, which we may assume by induction. Second, we may assume that the vertices in $S$ form a subpath of the base path of length $a$. Indeed, given the result in this case, we may write any $S$ as the disjoint union of subpaths $S_{1}, S_{2}, \ldots, S_{p}$ with no edges between them, so that

$$
e(S)=e\left(S_{1} \cup S_{2} \cup \cdots \cup S_{p}\right)=e\left(S_{1}\right)+e\left(S_{2}\right)+\cdots+e\left(S_{p}\right) \geq \rho_{T}\left(\left|S_{1}\right|+\left|S_{2}\right|+\cdots+\left|S_{p}\right|\right)=\rho_{T}|S| .
$$

Suppose, therefore, that $S=\{l, l+1, \ldots, r\}$ is a proper subpath of the base path $\{1,2, \ldots, a\}$ and $b-a=i$.

As the desired claim is trivially true if $i=-1$, we will assume that $i \geq 0$. In particular, it follows from this assumption that vertex 1 of the base path is adjacent to a rooted vertex.

Let $R$ be the number of rooted vertices adjacent to $S$. For $0 \leq j \leq i-1$, the $j$ th rooted vertex is adjacent to $S$ precisely when $l \leq 1+j\left(\frac{a}{i}\right)<r+1$, which is equivalent to

$$
(l-1) \frac{i}{a} \leq j<r \frac{i}{a}
$$

Therefore, if $a$ is not contained in $S$, it follows that $R \geq\left\lfloor|S| \frac{i}{a}\right\rfloor=\left\lfloor|S| \frac{b-a}{a}\right\rfloor$. Furthermore, if $l=1$, then $R=\left\lceil|S| \frac{b-a}{a}\right\rceil$. Finally, if $r=a$ and $i>0$, then, using

$$
a-\left\lfloor 1+j \cdot \frac{a}{i}\right\rfloor \leq(i-j) \frac{a}{i}
$$

it follows that $S$ is adjacent to the $j$ th root whenever $i|S| / a>i-j$, and so $R \geq\left\lceil|S| \frac{b-a}{a}\right\rceil$.

Case 1: $i=0$. Since $S$ is a proper subpath, it is adjacent to at least $|S|=(b / a)|S|$ edges.

Case 2: $R \geq\left\lceil|S| \frac{b-a}{a}\right\rceil$. Then the total number of edges adjacent to $S$ is at least $R+|S| \geq(b / a)|S|$.

Case 3: $i>0$ and $R<\left\lceil|S| \frac{b-a}{a}\right\rceil$. Then $S$ is adjacent to $|S|+1$ edges in the base path, for a total of $\left\lfloor|S| \frac{b-a}{a}\right\rfloor+|S|+1 \geq(b / a)|S|$ adjacent edges. 
Before moving on to the proof of Lemma 1.2, it will be useful to note that if $T$ is balanced then every graph in $\mathcal{T}^{p}$ is at least as dense as $T$.

Lemma 2.2 If $(T, R)$ is a balanced rooted tree, then every graph $H$ in $\mathcal{T}^{s}$ satisfies

$$
e(H) \geq \rho_{T}(|H|-|R|) .
$$

Proof: We will prove the result by induction on $s$. It is clearly true when $s=1$, so we will assume that it holds for any $H \in \mathcal{T}^{s}$ and prove it when $H \in \mathcal{T}^{s+1}$.

Suppose, therefore, that $H$ is the union of $s+1$ labelled copies of $T$, say $T_{1}, T_{2}, \ldots, T_{s+1}$, each of which agree on the set of roots $R$. If we let $H^{\prime}$ be the union of the first $s$ copies of $T$, the induction hypothesis tells us that $e\left(H^{\prime}\right) \geq \rho_{T}\left(\left|H^{\prime}\right|-|R|\right)$. Let $S$ be the set of vertices in $T_{s+1}$ which are not contained in $H^{\prime}$. Then, since $T$ is balanced, we know that $e(S)$, the number of edges in $T_{s+1}$ (and, therefore, in $H$ ) with at least one endpoint in $S$, is at least $\rho_{T}|S|$. It follows that

$$
e(H) \geq e\left(H^{\prime}\right)+e(S) \geq \rho_{T}\left(\left|H^{\prime}\right|-|R|\right)+\rho_{T}|S|=\rho_{T}(|H|-|R|),
$$

as required.

\subsection{The lower bound}

The proof of the lower bound will follow [4] and [5] quite closely. We begin by describing the basic setup and stating a number of lemmas which we will require in the proof. We will omit the proofs of these lemmas, referring the reader instead to [4] and [5].

Let $q$ be a prime power and let $\mathbb{F}_{q}$ be the finite field of order $q$. We will consider polynomials in $t$ variables over $\mathbb{F}_{q}$, writing any such polynomial as $f(X)$, where $X=\left(X_{1}, \ldots, X_{t}\right)$. We let $\mathcal{P}_{d}$ be the set of polynomials in $X$ of degree at most $d$, that is, the set of linear combinations over $\mathbb{F}_{q}$ of monomials of the form $X_{1}^{a_{1}} \cdots X_{t}^{a_{t}}$ with $\sum_{i=1}^{t} a_{i} \leq d$. By a random polynomial, we just mean a polynomial chosen uniformly from the set $\mathcal{P}_{d}$. One may produce such a random polynomial by choosing the coefficients of the monomials above to be random elements of $\mathbb{F}_{q}$.

The first result we will need says that once $q$ and $d$ are sufficiently large, the probability that a randomly chosen polynomial from $\mathcal{P}_{d}$ contains each of $m$ distinct points is exactly $1 / q^{m}$.

Lemma 2.3 Suppose that $q>\left(\begin{array}{c}m \\ 2\end{array}\right)$ and $d \geq m-1$. Then, if $f$ is a random polynomial from $\mathcal{P}_{d}$ and $x_{1}, \ldots, x_{m}$ are $m$ distinct points in $\mathbb{F}_{q}^{t}$,

$$
\mathbb{P}\left[f\left(x_{i}\right)=0 \text { for all } i=1, \ldots, m\right]=1 / q^{m} .
$$

We also need to note some basic facts about affine varieties over finite fields. If we write $\overline{\mathbb{F}}_{q}$ for the algebraic closure of $\mathbb{F}_{q}$, a variety over $\overline{\mathbb{F}}_{q}$ is a set of the form

$$
W=\left\{x \in \overline{\mathbb{F}}_{q}^{t}: f_{1}(x)=\cdots=f_{s}(x)=0\right\}
$$

for some collection of polynomials $f_{1}, \ldots, f_{s}: \overline{\mathbb{F}}_{q}^{t} \rightarrow \overline{\mathbb{F}}_{q}$. We say that $W$ is defined over $\mathbb{F}_{q}$ if the coefficients of these polynomials are in $\mathbb{F}_{q}$ and write $W\left(\mathbb{F}_{q}\right)=W \cap \mathbb{F}_{q}^{t}$. We say that $W$ has complexity 
at most $M$ if $s, t$ and the degrees of the $f_{i}$ are all bounded by $M$. Finally, we say that a variety is absolutely irreducible if it is irreducible over $\overline{\mathbb{F}}_{q}$, reserving the term irreducible for irreducibility over $\mathbb{F}_{q}$ of varieties defined over $\mathbb{F}_{q}$.

The next result we will need is the Lang-Weil bound [16] relating the dimension of a variety $W$ to the number of points in $W\left(\mathbb{F}_{q}\right)$. It will not be necessary to give a formal definition for the dimension of a variety, though some intuition may be gained by noting that if $f_{1}, \ldots, f_{s}: \overline{\mathbb{F}}_{q}^{t} \rightarrow \overline{\mathbb{F}}_{q}$ are generic polynomials then the dimension of the variety they define is $t-s$.

Lemma 2.4 Suppose that $W$ is a variety over $\overline{\mathbb{F}}_{q}$ of complexity at most $M$. Then

$$
\left|W\left(\mathbb{F}_{q}\right)\right|=O_{M}\left(q^{\operatorname{dim} W}\right) .
$$

Moreover, if $W$ is defined over $\mathbb{F}_{q}$ and absolutely irreducible, then

$$
\left|W\left(\mathbb{F}_{q}\right)\right|=q^{\operatorname{dim} W}\left(1+O_{M}\left(q^{-1 / 2}\right)\right) .
$$

We will also need the following standard result from algebraic geometry, which says that if $W$ is an absolutely irreducible variety and $D$ is a variety intersecting $W$, then either $W$ is contained in $D$ or its intersection with $D$ has smaller dimension.

Lemma 2.5 Suppose that $W$ is an absolutely irreducible variety over $\overline{\mathbb{F}}_{q}$ and $\operatorname{dim} W \geq 1$. Then, for any variety $D$, either $W \subseteq D$ or $W \cap D$ is a variety of dimension less than $\operatorname{dim} W$.

The final ingredient we require says that if $W$ is a variety which is defined over $\mathbb{F}_{q}$, then there is a bounded collection of absolutely irreducible varieties $Y_{1}, \ldots, Y_{t}$, each of which is defined over $\mathbb{F}_{q}$, such that $\cup_{i=1}^{t} Y_{i}\left(\mathbb{F}_{q}\right)=W\left(\mathbb{F}_{q}\right)$.

Lemma 2.6 Suppose that $W$ is a variety over $\overline{\mathbb{F}}_{q}$ of complexity at most $M$ which is defined over $\mathbb{F}_{q}$. Then there are $O_{M}(1)$ absolutely irreducible varieties $Y_{1}, \ldots, Y_{t}$, each of which is defined over $\mathbb{F}_{q}$ and has complexity $O_{M}(1)$, such that $\cup_{i=1}^{t} Y_{i}\left(\mathbb{F}_{q}\right)=W\left(\mathbb{F}_{q}\right)$.

We can combine the preceding three lemmas into a single result as follows:

Lemma 2.7 Suppose $W$ and $D$ are varieties over $\overline{\mathbb{F}}_{q}$ of complexity at most $M$ which are defined over $\mathbb{F}_{q}$. Then one of the following holds for all $q$ sufficiently large in terms of $M$ :

- $\left|W\left(\mathbb{F}_{q}\right) \backslash D\left(\mathbb{F}_{q}\right)\right| \geq q / 2$, or

- $\left|W\left(\mathbb{F}_{q}\right) \backslash D\left(\mathbb{F}_{q}\right)\right| \leq c$, where $c=c_{M}$ depends only on $M$.

Proof: By Lemma 2.6, there is a decomposition $W\left(\mathbb{F}_{q}\right)=\bigcup_{i=1}^{t} Y_{i}\left(\mathbb{F}_{q}\right)$ for some bounded-complexity absolutely irreducible varieties $Y_{i}$ defined over $\mathbb{F}_{q}$. If $\operatorname{dim} Y_{i} \geq 1$, Lemma 2.5 tells us that either $Y_{i} \subset D$ or the dimension of $Y_{i} \cap D$ is smaller than the dimension of $Y_{i}$. If $Y_{i} \subset D$, then the component does not contribute any point to $W\left(\mathbb{F}_{q}\right) \backslash D\left(\mathbb{F}_{q}\right)$ and may be discarded. If instead the dimension of $Y_{i} \cap D$ is smaller than the dimension of $Y_{i}$, the Lang-Weil bound, Lemma 2.4, tells us that for $q$ sufficiently large

$$
\left|W\left(\mathbb{F}_{q}\right) \backslash D\left(\mathbb{F}_{q}\right)\right| \geq\left|Y_{i}\left(\mathbb{F}_{q}\right)\right|-\left|Y_{i}\left(\mathbb{F}_{q}\right) \cap D\right| \geq q^{\operatorname{dim} Y_{i}}-O\left(q^{\operatorname{dim} Y_{i}-\frac{1}{2}}\right)-O\left(q^{\operatorname{dim} Y_{i}-1}\right) \geq q / 2 .
$$


On the other hand, if $\operatorname{dim} Y_{i}=0$ for every $Y_{i}$ which is not contained in $D$, Lemma 2.4 tells us that $\left|W\left(\mathbb{F}_{q}\right) \backslash D\left(\mathbb{F}_{q}\right)\right| \leq \sum\left|Y_{i}\left(\mathbb{F}_{q}\right)\right|=O(1)$, where the sum is taken over all $i$ for which $\operatorname{dim} Y_{i}=0$.

We are now ready to prove Lemma 1.2, that for any balanced rooted tree $(T, R)$ there exists a positive integer $p$ such that $\operatorname{ex}\left(n, \mathcal{T}^{p}\right)=\Omega\left(n^{2-1 / \rho_{T}}\right)$.

Proof of Lemma 1.2; Let $(T, R)$ be a balanced rooted tree with $a$ unrooted vertices and $b$ edges, where $R=\left\{u_{1}, \ldots, u_{r}\right\}$ and $V(T) \backslash R=\left\{v_{1}, \ldots, v_{a}\right\}$. Let $s=2 b r, d=s b, N=q^{b}$ and suppose that $q$ is sufficiently large. Let $f_{1}, \ldots, f_{a}: \mathbb{F}_{q}^{b} \times \mathbb{F}_{q}^{b} \rightarrow \mathbb{F}_{q}$ be independent random polynomials in $\mathcal{P}_{d}$. We will consider the bipartite graph $G$ between two copies $U$ and $V$ of $\mathbb{F}_{q}^{b}$, each of order $N=q^{b}$, where $(u, v)$ is an edge of $G$ if and only if

$$
f_{1}(u, v)=\cdots=f_{a}(u, v)=0 .
$$

Since $f_{1}, \ldots, f_{a}$ were chosen independently, Lemma 2.3 with $m=1$ tells us that the probability a given edge $(u, v)$ is in $G$ is $q^{-a}$. Therefore, the expected number of edges in $G$ is $q^{-a} N^{2}=N^{2-a / b}$.

Suppose now that $w_{1}, w_{2}, \ldots, w_{r}$ are fixed vertices in $G$ and let $C$ be the collection of copies of $T$ in $G$ such that $w_{i}$ corresponds to $u_{i}$ for all $1 \leq i \leq r$. We will be interested in estimating the $s$-th moment of $|C|$. To begin, we note that $|C|^{s}$ counts the number of ordered collections of $s$ (possibly overlapping or identical) copies of $T$ in $G$ such that $w_{i}$ corresponds to $u_{i}$ for all $1 \leq i \leq r$. Since the total number of edges $m$ in a given collection of $s$ rooted copies of $T$ is at most $s b$ and $q$ is sufficiently large, Lemma 2.3 tells us that the probability this particular collection of copies of $T$ is in $G$ is $q^{-a m}$, where we again use the fact that $f_{1}, \ldots, f_{a}$ are chosen independently.

Suppose that $H$ is an element of $\mathcal{T}_{\leq}^{s} \stackrel{\text { def }}{=} \mathcal{T}^{1} \cup \mathcal{T}^{2} \cup \cdots \cup \mathcal{T}^{s}$. Within the complete bipartite graph from $U$ to $V$, let $N_{s}(H)$ be the number of ordered collections of $s$ copies of $T$, each rooted at $w_{1}, \ldots, w_{r}$ in the same way, whose union is a copy of $H$. Then

$$
\mathbb{E}\left[|C|^{s}\right]=\sum_{H \in \mathcal{T}_{\leq}^{s}} N_{s}(H) q^{-a e(H)},
$$

while $N_{s}(H)=O_{s}\left(N^{|H|-|R|}\right)$. Since $(T, R)$ is balanced, Lemma 2.2 shows that $\frac{e(H)}{|H|-|R|} \geq \rho_{T}=\frac{b}{a}$ for every $H \in \mathcal{T}_{\leq}^{s}$. It follows that

$$
\begin{aligned}
\mathbb{E}\left[|C|^{s}\right] & =\sum_{H \in \mathcal{T}_{\leq}^{s}} N_{s}(H) q^{-a e(H)}=\sum_{H \in \mathcal{T}_{\leq}^{s}} O_{s}\left(N^{|H|-|R|}\right) q^{-a e(H)} \\
& =O_{s}\left(\sum_{H \in \mathcal{T}_{\leq}^{s}} q^{b(|H|-|R|)} q^{-a e(H)}\right)=O_{s}(1) .
\end{aligned}
$$

By Markov's inequality, we may conclude that

$$
\mathbb{P}[|C| \geq c]=\mathbb{P}\left[|C|^{s} \geq c^{s}\right] \leq \frac{\mathbb{E}\left[|C|^{s}\right]}{c^{s}}=\frac{O_{s}(1)}{c^{s}}
$$

Our aim now is to show that $|C|$ is either quite small or very large. To begin, note that the set $C$ is a subset of $X\left(\mathbb{F}_{q}\right)$, where $X$ is the algebraic variety defined as the set of $\left(x_{1}, \ldots, x_{a}\right) \in \overline{\mathbb{F}}_{q}^{b a}$ satisfying the equations

- $f_{i}\left(w_{k}, x_{\ell}\right)=0$ for all $k$ and $\ell$ such that $\left(u_{k}, v_{\ell}\right) \in T$ and 
- $f_{i}\left(x_{k}, x_{\ell}\right)=0$ for all $k$ and $\ell$ such that $\left(v_{k}, v_{\ell}\right) \in T$

for all $i=1,2, \ldots, a$. For each $i \neq j$ such that $v_{i}$ and $v_{j}$ are on the same side of the natural bipartition of $T$, we let

$$
D_{i j}=X \cap\left\{\left(x_{1}, \ldots, x_{a}\right): x_{i}=x_{j}\right\}
$$

and, for each $k, \ell$ such that $v_{k}$ and $u_{\ell}$ are on the same side of the bipartition, we let

$$
D_{k \ell}^{\prime}=X \cap\left\{\left(x_{1}, \ldots, x_{a}\right): x_{k}=w_{\ell}\right\}
$$

We put

$$
D \stackrel{\text { def }}{=} \bigcup_{i, j} D_{i j} \cup \bigcup_{k, \ell} D_{k \ell}^{\prime}
$$

The sets $D_{i j}$ and $D_{k \ell}^{\prime}$ capture those elements of $X$ which are degenerate and so not elements of $C$. As a union of varieties is a variety, the set $D$ is a variety that captures all degenerate elements of $X$. Furthermore, the complexity of $D$ is bounded since the number and complexity of the $D_{i j}$ and $D_{k \ell}^{\prime}$ is bounded.

By Lemma 2.7, we see that that there exists a constant $c_{T}$, depending only on $T$, such that either $|C| \leq c_{T}$ or $|C| \geq q / 2$. Therefore, by the consequence of Markov's inequality noted earlier,

$$
\mathbb{P}\left[|C|>c_{T}\right]=\mathbb{P}[|C| \geq q / 2]=\frac{O_{s}(1)}{(q / 2)^{s}} .
$$

We call a sequence of vertices $\left(w_{1}, w_{2}, \ldots, w_{r}\right)$ bad if there are more than $c_{T}$ copies of $T$ in $G$ such that $w_{i}$ corresponds to $u_{i}$ for all $1 \leq i \leq r$. If we let $B$ be the random variable counting the number of bad sequences, we have, since $s=2 b r$ and $q$ is sufficiently large,

$$
\mathbb{E}[B] \leq 2 N^{r} \cdot \frac{O_{s}(1)}{(q / 2)^{s}}=O_{s}\left(q^{b r-s}\right)=o(1)
$$

We now remove a vertex from each bad sequence to form a new graph $G^{\prime}$. Since each vertex has degree at most $N$, the total number of edges removed is at most $B N$. Hence, the expected number of edges in $G^{\prime}$ is

$$
N^{2-a / b}-\mathbb{E}[B] N=\Omega\left(N^{2-a / b}\right) .
$$

Therefore, there is a graph with at most $2 N$ vertices and $\Omega\left(N^{2-a / b}\right)$ edges such that no sequence of $r$ vertices has more than $c_{T}$ labelled copies of $T$ rooted on these vertices. Finally, we note that this result was only shown to hold when $q$ is a prime power and $N=q^{b}$. However, an application of Bertrand's postulate shows that the same conclusion holds for all $N$.

\section{Concluding remarks}

We have shown that for any rational number $r$ between 1 and 2, there exists a family of graphs $\mathcal{H}_{r}$ such that $\operatorname{ex}\left(n, \mathcal{H}_{r}\right)=\Theta\left(n^{r}\right)$. However, Erdös and Simonovits (see, for example, [7]) asked whether there exists a single graph $H_{r}$ such that $\operatorname{ex}\left(n, H_{r}\right)=\Theta\left(n^{r}\right)$. Our methods give some hope of a positive solution to this question, but the difficulties now lie with determining accurate upper bounds for the extremal number of certain graphs. 
To be more precise, given a rooted tree $(T, R)$, we define $T^{p}$ to be the graph consisting of the union of $p$ distinct labelled copies of $T$, each of which agree on the set of roots $R$ but are otherwise disjoint. Lemma 1.2 clearly shows that $\operatorname{ex}\left(n, T^{p}\right)=\Omega\left(n^{2-1 / \rho_{T}}\right)$ when $T$ is a balanced rooted tree. We believe that a corresponding upper bound should also hold.

Conjecture 3.1 For any balanced rooted tree $(T, R)$, the graph $T^{p}$ satisfies

$$
e x\left(n, T^{p}\right)=O_{p}\left(n^{2-1 / \rho_{T}}\right) .
$$

The condition that $(T, R)$ be balanced is necessary here, as may be seen by considering the graph in Figure 2, namely, a star $K_{1,3}$ with two rooted leaves. Then $T^{2}$ contains a cycle of length 4 , so the extremal number is $\Omega\left(n^{3 / 2}\right)$, whereas the conjecture would suggest that it is $O\left(n^{4 / 3}\right)$.

In order to solve the Erdös-Simonovits conjecture, it would be sufficient to solve the conjecture for the collection of rooted trees $T_{a, b}$ with $a<b$ and $(a, b)=1$. However, even this seems surprisingly difficult and the only known cases are when $a=1$, in which case $T$ is a star with rooted leaves and $T^{p}$ is a complete bipartite graph, or $b-a=1$, when $T$ is a path with rooted endpoints and $T^{p}$ is a theta graph.

Acknowledgements. We would like to thank Jacques Verstraete for interesting discussions relating to the topic of this paper. We would also like to thank an anonymous referee and Lisa Sauermann for a number of useful comments and corrections.

\section{References}

[1] N. Alon, L. Rónyai and T. Szabó, Norm-graphs: variations and applications, J. Combin. Theory Ser. B 76 (1999), 280-290.

[2] P. V. M. Blagojević, B. Bukh and R. Karasev, Turán numbers for $K_{s, t}$-free graphs: topological obstructions and algebraic constructions, Israel J. Math. 197 (2013), 199-214.

[3] W. G. Brown, On graphs that do not contain a Thomsen graph, Canad. Math. Bull. 9 (1966), $281-285$.

[4] B. Bukh, Random algebraic construction of extremal graphs, Bull. London Math. Soc. 47 (2015), 939-945.

[5] D. Conlon, Graphs with few paths of prescribed length between any two vertices, to appear in Bull. London Math. Soc.

[6] P. Erdös, On sequences of integers no one of which divides the product of two others and on some related problems, Mitt. Forsch.-Inst. Math. Mech. Univ. Tomsk 2 (1938), 74-82.

[7] P. Erdős, On the combinatorial problems which I would most like to see solved, Combinatorica 1 (1981), 25-42.

[8] P. Erdős, A. Rényi and V. T. Sós, On a problem of graph theory, Studia Sci. Math. Hungar. 1 (1966), 215-235. 
[9] P. Erdős and M. Simonovits, A limit theorem in graph theory, Studia Sci. Math. Hungar. 1 (1966), $51-57$.

[10] P. Erdős and A. H. Stone, On the structure of linear graphs, Bull. Amer. Math. Soc. 52 (1946), 1087-1091.

[11] R. J. Faudree and M. Simonovits, On a class of degenerate extremal graph problems, Combinatorica 3 (1983), 83-93.

[12] P. Frankl, All rationals occur as exponents, J. Combin. Theory Ser. A 42 (1986), 200-206.

[13] Z. Füredi and M. Simonovits, The history of degenerate (bipartite) extremal graph problems, in Erdős Centennial, 169-264, Bolyai Soc. Math. Stud., 25, Springer, Berlin, 2013.

[14] J. Kollár, L. Rónyai and T. Szabó, Norm-graphs and bipartite Turán numbers, Combinatorica 16 (1996), 399-406.

[15] T. Kővári, V. T. Sós and P. Turán, On a problem of K. Zarankiewicz, Colloq. Math. 3 (1954), $50-57$.

[16] S. Lang and A. Weil, Number of points of varieties in finite fields, Amer. J. Math. 76 (1954), 819-827. 